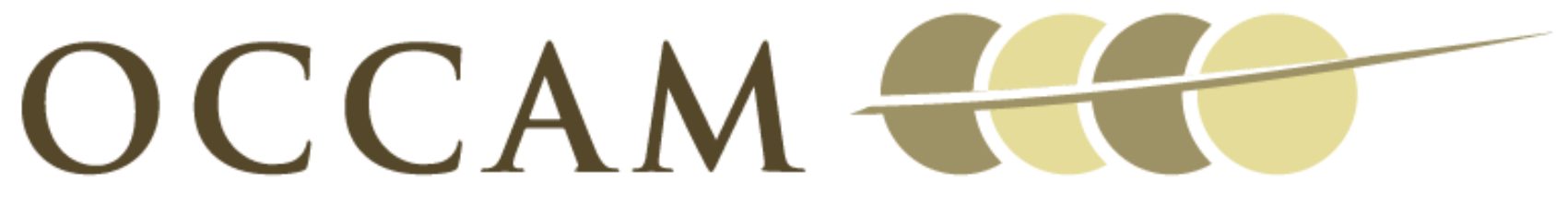

OXFORD CENTRE FOR COLLABORATIVE APPLIED MATHEMATICS

\author{
Report Number 10/19
}

Elastic cavitation, tube hollowing, and differential growth in plants and biological tissues

by

Alain Goriely, Derek E. Moulton and Rebecca Vandiver

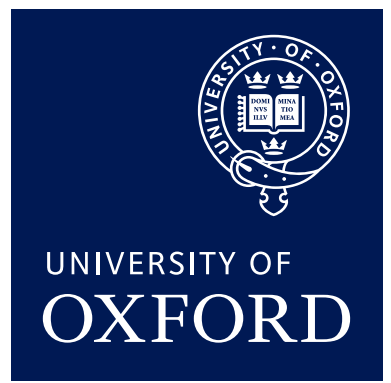

Oxford Centre for Collaborative Applied Mathematics Mathematical Institute 24 - 29 St Giles'

Oxford

OX1 3LB

England 



\title{
Elastic cavitation, tube hollowing, and differential growth in plants and biological tissues
}

\author{
Alain Goriely ${ }^{1}$, Derek E. Moulton ${ }^{1}$ and Rebecca Vandiver ${ }^{2}$ \\ 1 OCCAM Mathematical Institute, University of Oxford, UK \\ 2 Bryn Mawr College Bryn Mawr, PA 19010, USA
}

\author{
PACS 87.85.gp - Mechanical systems \\ PACS 46.32.+x - Static buckling and instability \\ PACS 89.20.-a - Interdisciplinary applications of physics
}

\begin{abstract}
Elastic cavitation is a well-known physical process by which elastic materials under stress can open cavities. Usually, cavitation is induced by applied loads on the elastic body. However, growing materials may generate stresses in the absence of applied loads and could induce cavity opening. Here, we demonstrate the possibility of spontaneous growth-induced cavitation in elastic materials and consider the implications of this phenomenon to biological tissues and in particular to the problem of schizogenous aerenchyma formation.
\end{abstract}

Introduction. - Among the many typical biological structures, tubular structures such as hollow stems and blood vessels abound in nature. Tubes are typically used for transport or mechanical support. Their morphogenesis usually involves complex genetic and biochemical process [Patan (2000)] mediated by mechanical forces. Here, we consider the possible role of mechanical stress in the opening of cavities in elastic tissues. Many biological tissues exhibit differential growth. That is, different parts of the tissue grow at different rates or in an anisotropic fashion. Typically these local changes of volume or mass induce elastic stresses that cannot be relieved by a change in geometry, hence building so-called residual stresses, stresses that remain in the body in the absence of body or external loads. Residual stresses are found universally in biological tissues and are the hallmark of mechanical biology. These stresses are known to play a role in the regulation of circumferential stress gradients in blood vessels [Fung (1991)], to improve the rigidity of growing plant stems [Vandiver and Goriely (2008)] and the stability of arteries [Goriely and Vandiver (2010)], and to assist in the proper functioning of airways and oesophagus [Han and Fung (1991)]. The theoretical analysis of residual stresses in growing elastic bodies has revealed that growth-induced stresses can trigger both mechanical instability [Goriely and Ben Amar( 2005)] and, in the case of elastic membranes, elastic cavitation [McMahon et al. (2008)].

A particularly striking example of cavity opening is found in the world of plants. Indeed, most people are

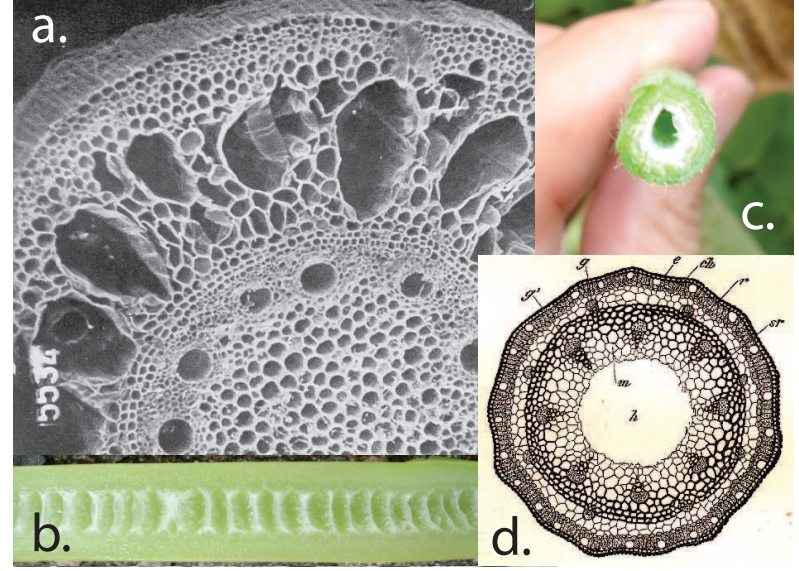

Fig. 1: A. Aerenchyma tissue in the roots of Zea mays, corn (from Kramer [Kramer (1983)]), B. Diaphragms in a sliced stem, C. Hollow stem in hollyhock Althaea rosea. D. Transverse section of the flowering scape of Allium Schoenoprasum (from Sachs [Sachs (1875)])

familiar with the simple observation that many plants such as dandelions, chives, and bamboo have hollow stems. Similarly, many roots, and water plants have tissues with large airy tissues known as aerenchyma (See Fig. 1). The existence of these pockets of gas in the plant tissues fulfill many functions such as mechanical economy and rigidity for hollow stems [Hoga and Niklas (1990)], buoyancy for hydrophytes, increase gas transport and sap flow, change 
in the scattering of light for chlorophyl production, and seed projection [Raven (1996)]. In the nineteenth century, Sachs [Sachs (1875),Newcombe (1894)] characterized the formation of aerenchyma as being either lysigenous, that is, created through uniform growth and cell death, or schizogenous, that is, air spaces are created through a process of differential growth creating tension in tissues that leads to the mechanical tearing of adjoining cells [Evans (2003)]. Schizogenous formation is associated with large and regular structures in plant tissues such as hollow stems of which Sachs write [Sachs (1875)]: "I will refer only to the one obvious fact that while the pith is no longer able to grow in proportion, it becomes ruptured while a cavity arises in the interior. This may be easily observed in the flower stems of the Teazel and Dandelion." While lysigenous aerenchyma formation has received considerable attention by plant physiologists [Kozela and Regan (2003)], schizogenous aerenchyma formation with its combined mechanical and developmental regulation is not well understood [Jackson and Armstrong (1999)] .

Schizogenous aerenchyma formation is a simple and beautiful example of the role that mechanics can play in the morphogenesis of long tubular structures. It naturally leads to the physical questions regarding the role of stresses and differential growth in the opening of cavities and in stem hollowing.

In the world on engineering and material sciences, elastic cavitation refers to the opening of voids in elastic materials. Following the early work of Gent and the seminal paper by Ball [Ball (1982), Cho et al. (1987)], it is well known that for a dead-load traction $p>p_{\mathrm{cr}}=5 E / 6$ on the outer boundary of an incompressible neo-Hookean sphere (where $E$ is the Young's modulus of infinitesimal deformations), the sphere supports the trivial spherical solution (with no cavity) and a cavitated solution with spherical symmetry whose cavity radius vanishes when $p=p_{\text {cr }}$. The existence of these two solutions meeting at $p=p_{\mathrm{cr}}$ and coexisting for $p>p_{\text {cr }}$ leads to the interesting (but somewhat controversial) possibility of a bifurcation between the trivial and cavitated state, with possible applications to void nucleation and fracture initiation [Antman (1995)] (see [Fond (2001)] for a review and some experimental verifications).

The purpose of this Letter is to study the general problem of elastic cavitation in residually stressed biological materials and to understand the role of mechanics in cavity and tube formation. We show that elastic cavitation can naturally occur as a result of the residual stress created during growth. The Letter is organized as follows. First, we consider elastic cavitation in elastic spherical shells growing differentially either anisotropically or inhomogeneously. Second, we look at the possibility of void opening in tubular structures modelled as cylindrical tubes and apply these ideas to the problem of hollowing in plant stems.

Morpho-elastic materials. - We first consider the growth of an incompressible hyperelastic spherical shell
[Pence and Tsai (2006)]. Let $\mathbf{x}=\boldsymbol{\chi}(\mathbf{X}, t)$ be the deformation of a three-dimensional elastic body where $\mathbf{X}$ and $\mathbf{x}$ describe the material coordinates of a point in the reference and current configurations. We use the theory of morpho-elasticity to describe deformation of a material that are due to the combination of both to and elasticity. Technically, by analogy with finite elasto-plasticity we introduce a multiplicative decomposition of the deformation gradient $\mathbf{F}=\operatorname{Grad}(\chi)$ to describe the growth process [Rodriguez et al. (1994)]. That is, we assume that $\mathbf{F}=\mathbf{A} \cdot \mathbf{G}$ is the product of a growth tensor $\mathbf{G}$, describing the growth of a local volume element, and an elastic tensor $\mathbf{A}$ which ensures compatibility and integrity during the growth process. Assuming that the material is hyperelastic, its response function is given by a strain energy density function $W=W(\mathbf{A})$ which sets the Cauchy stress tensor as

$$
\mathbf{T}=\mathbf{A} \cdot W_{\mathbf{A}}-p \mathbf{1}
$$

where $W_{\mathbf{A}}$ is the tensorial derivative of $W$ w.r.t. $\mathbf{A}$, and $p$ is associated with the incompressibility constraint [Ogden (1984)]. The Cauchy equations for the balance of linear and angular momenta are, in the static case,

$$
\operatorname{div}(\mathbf{T})=0 \text { and } \mathbf{T}^{\mathrm{T}}=\mathbf{T}
$$

where the divergence is taken in the current configuration. We assume that growth in the material takes place on a much slower time scale as the elastic response of the material so that the material can be considered at mechanical equilibrium at all time. For a given growth tensor or a constitutive law for the evolution of the growth tensor as a function of the other fields, these equations can be solved with suitable boundary conditions.

In the particular case of an initial incompressible sphere conserving its spherical symmetry during deformation and growth, these kinematic descriptors take a particularly simple form [Ben Amar and Goriely (2005)]

$$
\begin{aligned}
& \mathbf{F}=\operatorname{diag}\left(\partial_{R} r, r / R, r / R\right), \\
& \mathbf{A}=\operatorname{diag}\left(\alpha_{r}, \alpha_{\theta}, \alpha_{\varphi}\right)=\operatorname{diag}\left(\alpha^{-2}, \alpha, \alpha\right), \\
& \mathbf{G}=\operatorname{diag}\left(g_{r}, g_{\theta}, g_{\theta}\right)
\end{aligned}
$$

where the tensors are all expressed in the usual spherical coordinates $(R, \Theta, \Phi)$ and $(r, \theta, \phi)$ in the initial and current configuration. Let $a=r(A)$ and $b=r(B)$ be the inner and outer radii in the current configuration of a shell of initial radii $A$ ( $A=0$ for a sphere) and $B$. Once $a$ is known, the deformation is completely determined by the incompressibility condition

$$
r^{3}-a^{3}=3 \int_{A}^{R} R^{2} g_{r}(R) g_{\theta}^{2}(R) d R
$$

which also specifies the strain as a function of the reference radius

$$
\alpha=r(R) / R=\frac{1}{R}\left(a^{3}+3 \int_{A}^{R} R^{2} g_{r}(R) g_{\theta}^{2}(R) d R\right)^{1 / 3}
$$


After simplification, the radial component of the Cauchy stress is given by

$$
t_{r}(R)=t_{r}(A)+\int_{A}^{R} \frac{g_{r}}{g_{\theta} R \alpha^{2}} \partial_{\alpha} \widehat{W}(\alpha) d R,
$$

where $\alpha(R)=r(R) /\left(g_{\theta} R\right)$ and $\widehat{W}(\alpha)=W\left(\alpha^{-2}, \alpha, \alpha\right)$. In the absence of external loads, the boundary conditions are $t_{r}(B)=0$ and either $t_{r}(A)=0$ for a cavitated solution, or $t_{r}(0)$ finite for a sphere. Therefore, setting $R=B$ and $t_{r}(B)=0$, Eq. (8) together with Eq. (7) give an equation for a single parameter $a$

$$
0=t_{r}(A)+\int_{A}^{B} \frac{g_{r}}{g_{\theta} R \alpha^{2}} \partial_{\alpha} \widehat{W}(\alpha) d R .
$$

The problem of elastic cavitation is reduced to showing the existence of a solution with $a>0$. Before doing so, we find conditions for the existence of a trivial solution (that is, $a=0$, the solution with no inner cavity). Let $g_{1}=\lim _{R \rightarrow 0} g_{r}>0$ and $g_{2}=\lim _{R \rightarrow 0} g_{\theta}>0$ be the local growth elements at the origin. An expansion of (8) around $R=0$ reveals that

$$
t_{1}^{\prime}(R)=\frac{\left(g_{1} / g_{2}\right)^{1 / 3}}{R} \partial_{\alpha} \widehat{W}\left(\left(g_{1} / g_{2}\right)^{1 / 3}\right)+\mathrm{O}(1) .
$$

Since $t_{1}(R)$ has to be finite at the origin, we need $\partial_{\alpha} \widehat{W}\left(\left(g_{1} / g_{2}\right)^{1 / 3}\right)=0$ which is only guaranteed if $g_{1} / g_{2}=$ 1. We conclude that the trivial spherical solution ceases to exist unless $\lim _{R \rightarrow 0} g_{\theta} / g_{r}=1$; that is, close to the origin, growth must be isotropic for the trivial solution to persist.

Growth-induced cavitation in sphere. - We now study the possibility of growth-induced cavitation by looking at the existence of non-trivial solutions for given growth functions $g_{r}, g_{\theta}$. We consider two complementary cases, first the case of anisotropic but homogeneous growth and second, the case of isotropic but inhomogeneous growth. We restrict our attention to the neoHookean energy function

$$
W_{\mathrm{nh}}=\frac{\mu_{\mathrm{nh}}}{2}\left(\alpha_{r}^{2}+\alpha_{\theta}^{2}+\alpha_{\varphi}^{2}\right)-3
$$

(where we scale all forces by taking elastic modulus to be $\mu_{\mathrm{nh}}=1$, which corresponds for small deformation to a Young modulus $E=3$ ). In the first case (anisotropic growth), we take, without loss of generality $B=1$ and $g_{r}=1$ (that is a volume element grows along the two spherical angles and not along the radial direction, only the ratio $g_{\theta} / g_{r}$ plays a role in creating residual stress [Ben Amar and Goriely (2005)]). Then, Eq. (8), which can be integrated explicitly, provides an implicit relationship between $a$ and $g_{\theta}$. In Fig. 2, we graph the solution $a=a\left(g_{\theta}, A\right)$ for various initial inner radii. The cavitated solution from the sphere corresponds to the choice $A=0$. A local analysis of the solution close to $g_{\theta}=1$ leads to

$$
a \underset{g_{\theta} \rightarrow 1}{\sim} \sqrt{3} \exp \left[\frac{\sqrt{3} \pi}{18}-\frac{66 g_{\theta}^{2}+183 g_{\theta}-114}{36\left(5 g_{\theta}+1\right)\left(g_{\theta}-1\right)}\right],
$$

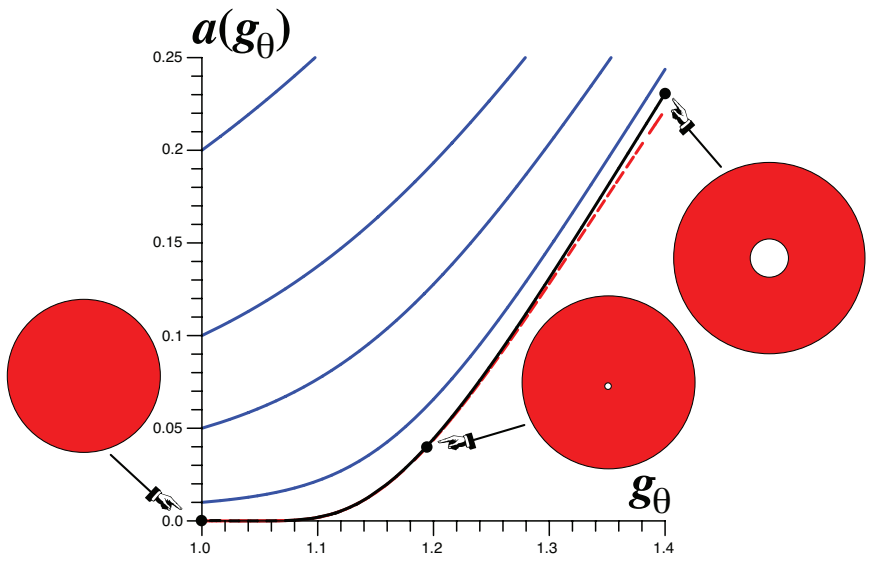

Fig. 2: Growth-induced cavitation of a sphere of initial radius 1 with constant anisotropic growth $\left(g_{r}=1, g_{\theta} \geq 1\right)$. Dashed curve: asymptotic solution tangent to the numerical solution. Solid curves correspond to cavity opening of a shell of inner radius $A=0.01,0.05,0.1,0.2$ due to growth.

which clearly establishes the existence of a cavitated solution whose inner radius is exponentially small close to $g_{\theta}=1$ but non-vanishing for all values of $g_{\theta}>1$. This is in contrast with the classical cavitation case for which the trivial solution persists and a large value of the external traction is required to obtain a cavitated solution. Physically, the two problems are different due to the particular boundary conditions associated with a residual stress field. The effect of anisotropic growth is twofold. First, the trivial spherical solution ceases to exist due to the local anisotropy at the origin. Second, the residual stress field creates radial tension close to the inner radius but satisfies the vanishing boundary condition for all values of $g_{\theta}$. In the classical case, a large elastic energy is necessary to balance a large gradient of radial stress close to the inner boundary which jumps from a finite value for the trivial solution to zero on the bifurcated solution.

In the second case (inhomogeneous growth), we set $g_{r}=g_{\theta}=1+\mu r$. Then, it can be similarly established that a cavity opens for $\mu>\mu_{c} \simeq 0.8971637$ as shown in Fig. 3. Here the radial tension at the origin increases with $\mu$ up to the critical point where sufficient elastic energy is built in the system to trigger cavitation. A similar mechanical environment could be created by considering an elastic sphere surrounded by a growing spherical shell. Again, for sufficiently large growth, the tension created by the shell pulling on the sphere would be enough to open a cavity. Therefore, we conclude that growth, either homogeneous or anisotropic, could be a simple and universal mechanism to open cavities in elastic materials. Its relevance to biological material is, however, still to be determined.

Before proceeding with the problem of stem hollowing, we should mention that there are two main fundamental issues related to both the classical case of elastic cavitation and the case of growth-induced cavitation. First, 

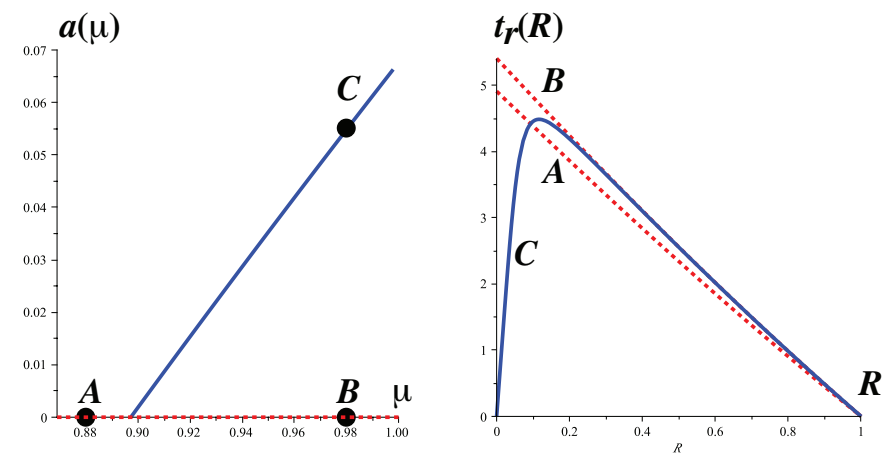

Fig. 3: Growth-induced cavitation of a sphere of initial radius 1 with isotropic homogeneous growth $\left(g_{r}=g_{\theta}=1+\mu R\right)$. Left: Opening $a$ as a function of $\mu$ after the critical value $\mu_{c}=$ 0.8971637 . Right: Radial tension as a function of initial radius $R$ for $\mu=0.88$ and $\mu=0.98$; dashed curve: trivial solution, solid curves, the cavitated solution with zero radial tension at the boundaries.

the possibility of a cavitation depends crucially on the choice of the strain-energy function. Namely, if we consider a perturbation of the neo-Hookean energy function $W=W_{\text {nh }}+\epsilon W_{\text {pert }}$ such that $\widehat{W}=\widehat{W}_{\text {nh }}+\epsilon \alpha^{n}$, then it is standard to show that there is no cavitated solution for all $n>3$ and $\epsilon \neq 0$ [Horgan and Polignone (1995)]; the bifurcation is topologically unstable as it disappears for arbitrarily small perturbations of the strain-energy function. Second, a similar cavitation analysis can be carried out in cylindrical geometry where one studies the bifurcation of a cylinder to a cylindrical tube. However, in this case, there is no possibility of cavitation for a neo-Hookean material (note however that Varga materials still exhibit cavitation [McMahon et al. (2008)]). This create a somewhat paradoxical situation if an anisotropic growth field is applied to a neo-Hookean cylinder: in this case, following the previous discussions, it is easy to show that neither the trivial solution nor a cavitated solution exists. In this case, either there is no solution to the morpho-elastic problem or there exists an an asymmetric solution (with or without a cavity); this remains an open problem.

The fact that cavitation depends both on the material response and on the particular geometry; that it changes the topology of the material; and that it relies on the assumption that elasticity remains valid at the microstructure suggests that cavitation may not be a robust material feature. However, cavitation is an idealized concept. If there exists a micro-void in the material, cavitation is replaced by the problem of cavity opening. As seen in Fig. 2, the opening of a small initial cavity at $A=0.01$ is essentially indistinguishable from the ideal cavitation problem and no experiment could distinguish between cavity opening and pure cavitation. Further, in the absence of a micro-void, the local stress field could be such that locally the material to reach the yield stress for rupture. At this point a small cavity opens due to tearing and the problem of ideal elastic cavitation can be replaced by the problem of the opening of a small cavity.

Plant stem opening. - We now consider an application of these ideas to the problem of cavity formation in plant stems. In a beautiful study, Takano et al. [Takano et al. (2001)] analyzed the effect of mechanical stress and gibberellins on stem hollowing in bean plants (see also [Carr and Jaffe (1995)]). Gibberellins are a well-known class of plant growth substances involved in stem elongation [Muto et al. (2004)]. In Takano's experiment, application of gibberellin to bush bean plants, which are naturally not hollow, increases the length of the stem, reduces its diameter, and induces stem hollowing . Conversely, in bean pole plants, which are naturally hollow, mechanical stresses [Jaffe and Forbes (1993), Pressman et al. (1983)] induced by rubbing cause an increase in the thickening, a reduction in axial length, and prevent stem hollowing.

While Takano's experiment suggests a connection between stem hollowing and differential growth, other authors have argued that cavity opening is the result of a chemical lysing process acting on the cell wall [Carr and Jaffe (1995)]. It is therefore of interest to study the possible role of mechanical stresses in this process. Here, we consider a simple model of stem growth where the stem is an incompressible neo-Hookean cylinder with initial outer radius $B$ subject to radial, angular, and axial deformation and growth along the three cylindrical coordinates $(r, \theta, z)$. As discussed above, cavitation is not possible for a neo-Hookean cylinder. Therefore, the idea is to explore whether growth induced stresses can be sufficient to induce rupture. Assuming that the cylinder retains its symmetry during deformation, the deformation, growth and elastic tensors are

$$
\begin{aligned}
& \mathbf{F}=\operatorname{diag}\left(r^{\prime}, r / R, \lambda_{z}\right), \\
& \mathbf{G}=\operatorname{diag}\left(g_{r}, g_{\theta}, g_{z}\right), \\
& \mathbf{A}=\operatorname{diag}\left(1 /\left(\alpha \alpha_{z}\right), \alpha, \alpha_{z}\right)
\end{aligned}
$$

The radial stress is then given by

$$
t_{r}(R)=t_{r}(0)+\int_{0}^{R} \frac{g_{r} g_{z} \hat{W}_{\alpha}}{\alpha_{z} g_{\theta} \alpha R} d R
$$

where $\widehat{W}(\alpha)=W\left(1 /\left(\alpha \alpha_{z}\right), \alpha, \alpha_{z}\right)$. This last equation with boundary conditions $t_{r}(B)=0$ and zero resultant load on the top and bottom [Rivlin (1949)], so that for a given growth tensor, the condition

$$
\int_{0}^{b} r t_{z}\left(r, \alpha_{z}\right) d r=0
$$

fixes the strains $\alpha$ and the tension at the origin, $t_{r}(0)$. Following known patterns of growth in stems [Peters et al. (2000)], we assume that on a given cross section, growth is isotropic but inhomogeneous by using a linear dependence with respect to the radius $R$. Further, we assume that axial growth creates tissue tension by being faster in the 
pith than the epidermis [Peters and Tomos (1996), Vandiver and Goriely (2008)]. That is,

$$
g_{r}=g_{\theta}=\nu_{1}+\nu_{2} R, \quad g_{z}=\mu_{1}+\mu_{2}(B-R) .
$$

Keeping the growth gradients $\nu_{2}$ and $\mu_{2}$ constant, an increase of the parameter $\mu_{1}$ corresponds to axial growth and an increase of of $\nu_{1}$ induces stem thickening which reproduce theoretically the changes in growth patterns corresponding to Takano's experiments. We compute the maximal stresses on the cylinder cross-section. The axial stress is always compressive due to the fact that the pith grows faster than the outer layers [Peters and Tomos (1996), Vandiver and Goriely (2008)]. However, both hoop and radial stresses at the origin $\left(t_{r}(0)\right.$ and $\left.t_{\theta}(0)\right)$ are equal and positive (i.e. tensile) for most realistic values of the growth parameters.


Fig. 4: Radial stress at the origin of a growing cylinder with constant inhomogeneous and anisotropic growth $\left(g_{r}=g_{\theta}=\right.$ $\left.\nu_{1}+\nu_{2} R, \quad g_{z}=\mu_{1}+\mu_{2}(B-R)\right)$ with parameter $\mu_{2}=2$. Left: the growth parameter $\nu_{1}=1$ is constant and the axial growth parameter $\mu_{1}$ is varied. Right: the axial growth parameter $\mu_{1}=10$ is constant and the constant sectional growth parameter is varied. The dashed line corresponds to an estimate of the breaking strength (See text). The material is assumed to be elastic with a strain-energy function $W_{\mathrm{nh}}$.

In Fig. 4, we plot this maximal value as a function of the axial and sectional growth parameters $\left(\mu_{1}\right.$ and $\nu_{1}$ respectively). In addition, we plot the value of breaking stress for pith tissue estimated from [Niklas (1993)] in which the Young Modulus for the pith is around 1 Mpa (Fig. 1 in [Niklas (1993)]) and values of the breaking stresses are around 0.3 MPa (Fig. 2 in [Niklas (1993)]). Since we have rescaled the stresses by setting the Young modulus $E=3$, the breaking stress is around $t_{\text {break }}=1$ in rescaled variables.

This analysis shows that the sign (compressive or tensile) and magnitude of the mechanical stresses acting on the cross-section of a stem are consistent with the hypothesis that aerenchyma is the result of mechanical tearing (note however that in similar experiments on celery and tomato pithiness, Jaffe and co-workers have argued that chemical lysis play the dominant-if not unique- role in aerenchyma). This tearing could be further enhanced by an underlying chemical process either weakening the pectin in the middle lamina or by the opening of the tricellular junction [Jarvis (1998), Jarvis et al. (2003)].

We have shown that cavity opening in elastic tissues can be induced by either anisotropic or inhomogeneous growth even in the absence of external tractions. However, continuum mechanics is topologically unstable and potentially paradoxical with regards to pure cavitation. A detailed study of the stresses generated in the growth of plant stems reveals that residual stresses likely provide the resolution, creating a mechanical environment suitable for tissue tearing, beyond which stem hollowing involves the robust elastic process of the opening of a cavity.

Acknowledgments: The authors would like to thank Prof. D. Cosgrove for helping them locate relevant literature on stem hollowing. This publication is based on work supported by Award No. KUK-C1-013-04 , made by King Abdullah University of Science and Technology (KAUST), and based in part upon work supported by the National Science Foundation under grants DMS-0907773 (AG). AG is a Wolfson/Royal Society Merit Award Holder.

\section{REFERENCES}

[Patan (2000)] S. Patan, J. Neuro-Oncology 50 (2000).

[Fung (1991)] Y. C. Fung, Annals of Biomedical engineering 19, 237 (1991).

[Vandiver and Goriely (2008)] R. Vandiver and A. Goriely, Europhys. Lett. (EPL) 84 (2008).

[Goriely and Vandiver (2010)] A. Goriely and R. Vandiver, IMA J. Appl. Math. Accepted (2010).

[Han and Fung (1991)] H. C. Han and Y. C. Fung, J Biomech 24, 307 (1991).

[Goriely and Ben Amar( 2005)] A. Goriely and M. Ben Amar, Phys. Rev. Lett. 94, 198103 (2005), ISSN 0031-9007 (Print).

[McMahon et al. (2008)] J. McMahon, A. Goriely, and M. Tabor, Mathematics and Mechanics of Solids p. doi:10.1177/1081286508092010 (2008).

[Kramer (1983)] P. J. Kramer, Water Relations of Plants (Academic Press, Inc. Orlando, FL., 1983).

[Sachs (1875)] J. Sachs, Text-book of botany, morphological and physiological (Clarendon, Oxford, 1875).

[Hoga and Niklas (1990)] C. J. J. Hoga and K. J. Niklas, American Journal of Botany 90, 356 (1990).

[Raven (1996)] J. A. Raven, Annals of Botany 78, 137 (1996).

[Newcombe (1894)] F. C. Newcombe, Ann. Bot. Lond. 8, 403 (1894).

[Evans (2003)] D. E. Evans, New Phytologist 161, 35 (2003).

[Kozela and Regan (2003)] C. Kozela and S. Regan, Trends Plant Sci 8, 159 (2003), ISSN 1360-1385 (Print).

[Jackson and Armstrong (1999)] M. B. Jackson and W. Armstrong, Plant Biol. 1, 274 (1999).

[Ball (1982)] J. M. Ball, Phil. Trans. R. Soc. London A 306, 557 (1982), ISSN 0080-4614.

[Cho et al. (1987)] K. Cho, A. N. Gent, and P. S. Lam, J. Mat. Sci. 22, 2899 (1987).

[Antman (1995)] S. S. Antman, Nonlinear problems of elasticity (Springer Verlag, New York, 1995). 
[Fond (2001)] C. Fond, J. Polymer Sci. B: Polymer Phys. 39, 2081 (2001).

[Pence and Tsai (2006)] T. Pence and H. Tsai, Math. Mech. Solids 11, 527 (2006).

[Rodriguez et al. (1994)] E. K. Rodriguez, A. Hoger, and A. McCulloch, J. Biomechanics 27, 455 (1994).

[Ogden (1984)] R. W. Ogden, Non-linear elastic deformation (Dover, New york, 1984)

[Ben Amar and Goriely (2005)] M. Ben Amar and A. Goriely, J. Mech. Phys. Solids 53, 2284 (2005).

[Horgan and Polignone (1995)] C. . Horgan and D. A. Polignone, Appl. Mech. Rev. 48, 471 (1995).

[Takano et al. (2001)] M. Takano, H. Takahashi, and H. Suge, Plant and Cell Physiology 36, 101 (2001).

[Carr and Jaffe (1995)] S. Carr and M. Jaffe, Annals of Botany 75, 587 (1995).

[Muto et al. (2004)] H. Muto, N. Yabe, T. Asami, K. Hasunuma, and K. Yamamoto, Plant Physiology (2004).

[Jaffe and Forbes (1993)] M. Jaffe and S. Forbes, Plant Growth Regulation 12, 313 (1993).

[Pressman et al. (1983)] E. Pressman, M. Huberman, B. Alaoni, and M. J. Jafe, Annals of Botany 52, 93 (1983)

[Rivlin (1949)] R. S. Rivlin, Phil. Trans. R. Soc. London A 242, 173 (1949).

[Peters et al. (2000)] W. Peters, W. Hagemann, and D. A. Tomos, Comparative Biochemistry and Physiology, Part A 125, 151 (2000).

[Peters and Tomos (1996)] W. S. Peters and A. D. Tomos, Ann Bot (Lond) 77, 657 (1996).

[Niklas (1993)] K. J. Niklas, Ann. Bot. Lond. 72, 173 (1993).

[Jarvis (1998)] M. C. Jarvis, Plant Cell and Environment 21, 1307 (1998).

[Jarvis et al. (2003)] M. C. Jarvis, S. P. H. Briggs, and J. P. Knox, Plant Cell and Environment 26, 977 (2003). 


\section{RECENT REPORTS}

43/09 Pseudoreplication invalidates the results of many neuroscientific Lazic studies

44/09 Cardiac cell modelling: Observations from the heart of the cardiac physiome project

45/09 A Hybrid Radial Basis Function - Pseudospectral Method for Thermal Convection in a 3-D Spherical Shell

Wright

Flyer

46/09 Refining self-propelled particle models for collective behaviour Yates

Baker

Erban

Maini

47/09 Stochastic Partial Differential Equations as priors in ensemble methods for solving inverse problems

Potsepaev

Farmer

Aziz

48/09 DifFUZZY: A fuzzy spectral clustering algorithm for complex data sets

Cominetti et al.

01/10 Fluctuations and instability in sedimentation

Guazzelli

Hinch

02/10 Determining the equation of state of highly plasticised metals from Hinch boundary velocimetry

03/10 Stability of bumps in piecewise smooth neural elds with nonlinear adaptation

Kilpatrick

Bressloff

04/10 Random intermittent search and the tug-of--war model of motordriven transport

Newby

Bressloff

05/10 Ergodic directional switching in mobile insect groups

Escudero et al.

06/10 Derivation of a dual porosity model for the uptake of nutrients by root hairs

Zygalakis

Roose

07/10 Frost heave in compressible soils

Majumdar

Peppin

Style

Sander

08/10 A volume-preserving sharpening approach for the propagation of

Reis sharp phase boundaries in multiphase lattice Boltzmann simulations

Dellar 
09/10 Anticavitation and differential growth in elastic shells Moulton

Goriely

10/10 On the mechanical stability of growing arteries Goriely

Vandiver

11/10 Nonlinear Correction to the Euler Buckling Formula for Com- De Pascalis pressible Cylinders

Destrade

Goriely

12/10 Nonlinear Morphoelastic Plates I: Genesis of Residual Stress McMahon

Goriely

Tabor

13/10 Nonlinear Morphoelastic Plates II: Exodus to Buckled States

McMahon

Goriely

Tabor

14/10 Analysis of Brownian dynamics simulations of reversible biomolecular reactions

Lipkova

Zygalakis

Chapman

Erban

15/10 Travelling waves in hyperbolic chemotaxis equations

Xue

Hwang

Painter

Erban

16/10 The Physics and Mechanics of Biological Systems

Goriely

Moulton

17/10 Crust formation in drying colloidal suspensions

Style

Peppin

18/10 A Mathematical Model of Tumor-Immune Interactions

Robertson-Tessi

El-Kareh

Goriely

Copies of these, and any other OCCAM reports can be obtained from:

Oxford Centre for Collaborative Applied Mathematics Mathematical Institute

24 - 29 St Giles'

Oxford

OX1 3LB

England

www.maths.ox.ac.uk/occam 